EXEMPLARIa Classica

Journal of Classical Philology

21, 2017, pp. 227-242

ISSN 1699-3225

\title{
SCHOLIA IN SCHOLIA: SU UNA NUOVA EDIZIONE DI HERMIAS DI ALESSANDRIA
}

Carlo M. Lucarini et Claudio Moreschini, Hermias Alexandrinus, In Platonis Phaedrum scholia, Berlin - Boston: De Gruyter, 2012, lxiv+293 pp., ISBN 978-3-11-020115-4. ${ }^{1}$

Negli ultimi anni l'interesse verso i più tardi filosofi Neoplatonici, per la verità mai del tutto sopito, ha fatto registrare un sensibile incremento, particolarmente notevole soprattutto in Francia, dove, grazie all'impulso del compianto Alain-Philippe Segonds (1942-2011), direttore generale de Les Belles Lettres sin dal 1988, sono stati dedicati ad essi molti volumi della Collection des Universités de France, tutti dotati di corpose introduzioni, testo fondato criticamente, traduzioni in francese e, soprattutto, ampli, anzi amplissimi apparati di Notes complémentaires. ${ }^{2}$ Così, per limitarci ai soli commentarî a Platone, hanno visto la luce quelli di Proclo all'Alcibiade I (1985-1986) e al Parmenide (2007-), entrambi a cura di Segonds stesso, con la collaborazione di Concetta Luna per il secondo, ${ }^{3}$ e quelli di Damascio al Filebo (2008), di Gerd Van Riel, e al Parmenide (1997-2003), èdito da Leendert Gerrit Westerink (1913-1990), con la collaborazione di Joseph Combès (1920-2002), Segonds, nonché Concetta Luna per l'ultimo volume. ${ }^{4}$ Del commentario di Proclo al Parmenide esiste anche un'altra recentissima edizione, coordinata da Carlos Steel e pubblicata per la serie Scriptorum Classicorum Bibliotheca Oxoniensis fra 2007 e $2009 .{ }^{5}$

${ }^{1} \mathrm{Nel}$ seguito, indicheremo i due autori con le lettere iniziali dei loro cognomi ed entrambi con la sigla L.-M.

${ }^{2}$ Sui progetti editoriali di Segonds e sul suo impulso in favore dei testi Neoplatonici, rimando al ricordo che ne ha scritto H. D. Saffrey, "Prologue", Studia Graeco-Arabica 2, 2012, vii-ix.

${ }^{3}$ A.-Ph. Segonds, Proclus. Sur le Premier Alcibiade de Platon, 2 voll., Paris 1985-1986; Concetta Luna - A.-Ph. Segonds, Proclus. Commentaire sur le Parménide de Platon, 5 vol., Paris 2007-2014. Segonds ci ha purtroppo lasciati poco tempo prima della pubblicazione del terzo volume dell'In Parmenidem, nel 2011, ma Concetta Luna ne sta portando avanti la pubblicazione.

${ }^{4}$ G. Van Riel, Damascius. Commentaire sur le Philèbe de Platon, Paris 2008; L. G. Westerink - J. Combès - A.-Ph. Segonds - C. Luna, Damascius. Commentaire sur le Parménide de Platon, 4 vol., Paris 1997-2003. C’è da precisare che dell'In Philebum esisteva già l'eccellente edizione di L. G. Westerink, Damascius. Lectures on the Philebus, wrongly attributed to Olympiodorus, Amsterdam $1959\left(=1982^{2}\right)$.

${ }^{5}$ C. Steel, Procli in Platonis Parmenidem commentaria, 3 voll., Oxford 2007-2009. 
Per gli altri commentarî neoplatonici, dobbiamo ricorrere ad edizioni critiche più datate, risalenti all'inizio del Novecento. In particolare, negli anni 1899-1901 videro la luce quelli alla Repubblica, curati da Wilhelm Kroll (1869-1939), ${ }^{6}$ nel 1903-1906 quello al Timeo di Ernst Diehl (1874-1947) ${ }^{7}$ e nel 1908 quello al Cratilo, pubblicato da Giorgio Pasquali (1885-1952). ${ }^{8}$ Alla seconda metà del medesimo secolo appartengono invece quello di Damascio a Fedone (1977) e quelli di Olimpiodoro ad Alcibiade I (1954), Gorgia (1970) e Fedone (1976), tutti a cura di Westerink. ${ }^{9}$

Curiosamente escluso dalla serie di edizioni o riedizioni moderne rimaneva Hermias, ${ }^{10}$ il filosofo nativo di Alessandria, allievo, ad Atene, insieme all'amico Proclo, di Siriano, di cui sposò pure una parente, Edesia. ${ }^{11}$ Di lui ci resta un commento al Fedro in tre libri, ${ }^{12}$ l'unico a noi giunto dall'antichità dedicato a questo dialogo platonico ${ }^{13}$ tramandato sotto il titolo di

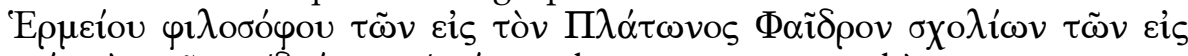

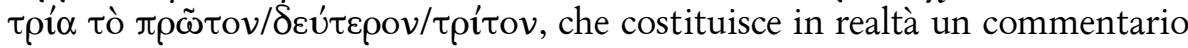
del tipo ó̀ंò $\varphi \omega v \tilde{\eta} \varsigma,{ }^{14}$ quasi certamente derivato dalle lezioni di Siriano. Il

${ }^{6}$ W. Kroll, Procli Diadochi in Platonis Rem Publicam commentarii, 2 voll., Leipzig
1899-1901.
${ }^{7}$ E. Diehl, Procli Diadochi In Platonis Timaeum commentaria, 3 voll., Leipzig 19031906.

${ }^{8}$ G. Pasquali, Procli in Platonis Cratylum commentaria, Leipzig 1908.

${ }^{9}$ L. G. Westerink, Proclus Diadochus. Commentary on the first Alcibiades of Plato, Amsterdam $1954\left(=1982^{2}\right)$; Id., Olympiodori in Platonis Gorgiam commentaria, Leipzig 1970; Id., The Greek commentaries on Plato's Phaedo, vol. 1. Olympiodorus, Amsterdam - Oxford - New York 1976; Id., The Greek commentaries, vol. 2. Damascius, Amsterdam - Oxford - New York 1977. Per dovere di cronaca, si deve segnalare che dei due commenti di Olimpiodoro esistevano delle precedenti edizioni, curate da William Norvin (1878-1940): Olympiodori philosophi in Platonis Phaedonem commentaria, Leipzig 1913; Olympiodori Philosophi in Platonis Gorgiam Commentaria, Leipzig 1936.

${ }^{10} \mathrm{Si}$ deve però segnalare una eccellente traduzione in tedesco, dotata di un ampio apparato di note: H. Bernard, Hermeias von Alexandrien. Kommentar su Platons "Phaidros", Tübingen 1997.

${ }^{11}$ Per una biografia sull'autore, rimando naturalmente a: K. Praechter, $R E$, s. v. "Hermeias 13", 15, 1912, 732-735; Bernard, Hermeias von Alexandrien, 1-4; R. Goulet, "Hermeias d'Alexandrie", in R. Goulet (ed.), Dictionnaire des Philosophes Antiques, III, Paris 2000, 639-641.

${ }^{12}$ Sappiamo anche di un suo testo matematico contenuto in un codice Scorial. gr. E III 8, perduto a causa dell'incendio che nel 1671 devastò la biblioteca.

${ }^{13}$ H. Dörrie - M. Balthes, Der Platonismus im 2. und 3. Jahrhundert nach Christus, 3, Stuttgart - Bad Cannstatt 1993, 197-198, in rapporto ai frr. 79.3a e 79.3b, menzionano, fra i Neoplatonici, commenti di Giamblico, Porfirio, Teodoro di Asine, Siriano e Proclo. La fortuna del dialogo platonico presso i Neoplatonici è ben documentata dal fatto che esso, secondo il catalogo di Giamblico, doveva essere letto come ottavo, e dunque verso la fine del primo ciclo di dieci dialoghi: cf. ex. gr. L. G. Westerink - J. Trouillard - A.-Ph. Segonds, Prolégomènes à la Philosophie de Platon, Paris 1990, lxvii-lxxiv.

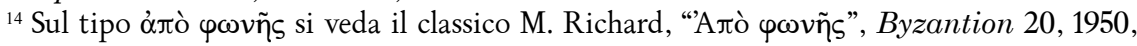
191-222. 
termine $\sigma \chi \chi^{\prime} \lambda_{1} \alpha$ del titolo, anziché l'atteso $\sigma \chi 0 \lambda \alpha i,{ }^{15}$ allude probabilmente alla struttura dell'opera, in effetti caratterizzata da commenti più o meno estesi dedicati all'esegesi di pericopi testuali platoniche di cui l'autore (o il redattore) riproduce le prime parole. ${ }^{16}$

Di quest'opera, l'edizione di riferimento è stata fino ad ora quella curata da Paul Couvreur e pubblicata postuma da Louis Bodin nel $1901 .{ }^{17}$ Si trattava e, per la verità, si tratta ancor oggi, di una edizione di altissimo pregio, sia per l'estremamente accurata collazione del codice Par. gr. 1810 (A), che gli studi più recenti avevano già indicato come codex unicus ${ }^{18}$ sia per la qualità e l'affidabilità complessiva del testo greco ivi pubblicato. Il limite principale consisteva nella conoscenza della tradizione manoscritta, che non andava molto al di là dei codici Parigini, a lui meglio accessibili: in prefazione (p. $x-x v i)$ elenca infatti solo 30 testimoni, più altri cinque non meglio identificati, e comunque a lui ignoti o del tutto perduti, e anche lo stemma codicum da lui proposto alla pag. xvii appare decisamente molto sommario.

Couvreur era ben consapevole dell'incompletezza del suo catalogo di codici (cf. ex. gr. p. xvi: «et certo nonnullos omisi»), ma nessuno studioso aggredì la questione dopo di lui, se non Maria Elisabetta Minniti Colonna, che nel 1978 rese noti e descrisse accuratamente cinque manoscritti napoletani, dei quali solo uno era in qualche modo noto all'editore francese. ${ }^{19}$ Infine, nel 2007, un fondamentale contributo alla conoscenza anche di Hermias venne dalla pubblicazione del primo volume della succitata edizione, curata da Concetta Luna e Alain-Philippe Segonds, dell'In Parmenidem di Proclo, la cui opera è spesso trasmessa nei codici insieme a quella del compagno di scuola e amico.

${ }^{15}$ Il termine $\sigma x o \lambda \alpha i$ fa riferimento alle lezioni di un dato autore, ad esempio un filosofo, spesso raccolte e pubblicate da qualche allievo, mentre con ơó $\lambda_{1} \alpha$ ci si riferisce proprio alle piccole note e ad osservazioni puntuali su una singola questione: cf. G. Zuntz, Die Aristophanes-Scholien der Papyri, Berlin 1975², 65-67, che però non menziona la nostra opera.

${ }^{16} \mathrm{~J}$. M. Dillon, Iamblichi Chalcidensis in Platonis Dialogos Commentariorum Fragmenta, Leiden 1973, 63.

${ }^{17}$ P. Couvreur, Hermiae Alexandrini in Platonis Phaedrum scholia ad fidem codicis Parisini denuo collati edidit et apparatu critico ornavit, Paris 1901 (ristampato a cura di C. Zintzen, Hildesheim - New York, 1971). La precedente edizione, vera editio princeps, era stata quella che nel 1810 aveva curato Georg Anton Friedrich Ast (Platonis Phaedrus recensuit, Hermiae scholiis e cod. Monac. XI suisque commentariis illustravit, Lipsiae 1810), ma essa si basava su un solo codice, il Monac. gr. 11 (M), del 1549 e ovviamente secondario. Alle pag. Ixi-lxii della prefazione della nostra edizione, vengono anche elencati gli estratti dell'opera precedentemente pubblicati, risalendo al De patria Homeri di Leone Allacci, stampato a Lione nel 1640 .

${ }^{18}$ Il merito spetta a A(lbrecht) Jordan, "Zur Kritik der spätern Platoniker", Hermes 14, 1879, 262-268 (si noti che L.-M. lo indicano con il nome «H(einrich) Jordan», confondendolo con un altro studioso in quegli anni molto attivo sulla stessa rivista; l'articolo immediatamente successivo è proprio di costui).

${ }^{19}$ M. Minniti Colonna, "I codici napoletani degli «Scholia in Platonis Phaedrum» di Ermia di Alessandria", Vichiana 7, 1978, 193-201. 
Probabilmente anche sulla scia dell'In Parmenidem procliano, Claudio Moreschini, già editore del Fedro platonico e autore di contributi specifici su Hermias almeno sin dai primi anni ' $90,{ }^{20}$ ha deciso di preparare una nuova edizione dell'In Phaedrum, avvalendosi della collaborazione di un più giovane, ma già allora affermatissimo studioso come Carlo Martino Lucarini. ${ }^{21}$ Entrambi hanno curato il testo (le singole congetture sono attribuite al rispettivo autore) e il capitolo De librorum necessitudinibus (p. xxxiiixxxix) della prefazione; il solo Lucarini è autore di tutto il resto della prefazione e della relativa appendice.

Inutile precisare che la nuova edizione rappresenta un enorme passo in avanti. Il catalogo dei manoscritti (cap. Codicum laterculum), che integra e arricchisce precedenti lavori di L., ${ }^{22}$ porta il totale a 42 , ai quali ne vanno aggiunti altri tre oggi perduti e la traduzione di Marsilio Ficino, a sua volta tramandata da due codici. Di questi testimoni, ben 12 e la traduzione, tutti segnalati dall'editore con un asterisco, erano ignoti a Couvreur. Su ciascuno dei manoscritti vengono fornite rapide indicazioni, eccezion fatta per il Par. Gr. 1810, cui sono dedicate più pagine. In questa sezione ho rinvenuto

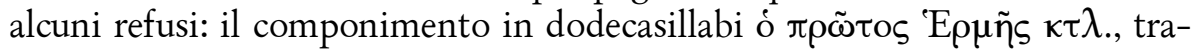
scritto a p. x, non è attestato al f. 93v, ma sul margine inferiore del f. 93r, proprio alla fine del testo di Hermias; alla nota 28 pag. xii, citando le parole di Luna - Segonds, si scrive "cette restauration du ms. A (in grassetto nel testo) été exécutée...», ma naturalmente doveva essere "a été exécutée»; del ms. Oxoniensis Bodleianus Auct. F.1.1 (p. xvi) è stata omessa l'indicazione della datazione; ${ }^{23}$ alla p. xxiii, nella sezione del codice Ven. Marc. gr. 228, «Mos^in» anziché «Mošin».

I capitoli successivi della prefazione sono dedicati all'indagine sulla tradizione indiretta, estremamente importante nel caso del nostro autore, poiché tutti i testimoni sono più antichi del codice $\mathrm{A}$.

${ }^{20}$ Le edizioni del Fedro platonico: C. Moreschini, Platonis Parmenides, Phaedrus, Roma 1966; Id. Platon, Phèdre, Paris 1985. Il suo primo contributo a me noto sull'autore è "Alcuni aspetti degli Scholia in Phaedrum di Ermia Alessandrino”, in Marie-Odile Goulet-Cazé, Goulven Madec e Denis O'Brien (edd.), $\Sigma$ ОФIH $\Sigma$ MAIHTOPE $\Sigma$ «chercheurs de sagesse», Hommage à J. Pepin, Paris 1992, 451-460.

${ }^{21}$ Già autore di ben tre edizioni critiche: Herodianus, Regnum post Marcum, München/ Leipzig 2005; Paulini Pellaei Carmina. Accedunt duo carmina ex cod. Vat. Urb. 533, München/Leipzig 2006; Q. Curtius Rufus, Historiae, Berlin/New York 2009. In ambito neoplatonico, segnalo anche il suo "Per il testo di Alessandro di Afrodisiade e di Proclo", Orpheus n. s. 28, 2007, 128-137.

${ }^{22}$ C. M. Lucarini, "De Hermiae commentariorum in Phaedrum codicibus quaestiones", Hermes 140, 2012, 71-88; Id., "Epimetrum de Hermiae codicibus”, Hermes 141, 2013, 244-248.

${ }^{23} \mathrm{Il}$ codice è databile alla metà del XVI secolo, poiché le pagine contenenti Hermias sono di mano di Giovanni Mourmouris (RGKI 172), la cui attività si snoda fra 1550 e 1563: cf. Lucarini, "De Hermiae", 75. 
I primi ad essere oggetto di indagine sono gli scolî platonici, tramandati, come è noto, da manoscritti ben più antichi rispetto al Parigino di Hermias. ${ }^{24}$ Dopo aver segnalato una manciata di casi in cui essi riportano un testo migliore di quello di A (p. xxii), l'indagine si diffonde sugli scolî al Fedro pubblicati da Siebenkees e rièditi da L. in appendice alla prefazione (pp. liii-lx). ${ }^{25}$ Essi contengono, tra l'altro, una serie di estratti da Hermias che non fanno parte del corpus degli scolî platonici, anche se uno di essi era ancora considerato tale dai vecchi editori. ${ }^{26} \mathrm{~L}$. giustamente conclude che si tratta di meri estratti da Hermias, per errore pubblicati come scolî platonici, ${ }^{27}$ sia perché, come ricorda L., "nemo facile concedat librum Platonicum amplioribus scholiis auctum saec. XVIII in bibliotheca Marciana vel Angelica extitisse» (p. $\mathrm{xxx}),{ }^{28}$ sia perché, come mi permetterei di aggiungere, la confusione può essere stata favorita anche dalle modalità di trasmissione del testo del commentario: si ricordi infatti che Hermias è in genere tramandato accanto al testo platonico e addirittura nel codice A esso circonda su tre lati, a formare una vera e propria corona di scolî, il Fedro. ${ }^{29}$ La successiva ipotesi che Siebenkees abbia contaminato il codice 1 (Laur. Conv. Sop. 78) ed un rappresentante non meglio identificato della famiglia $\rho$, formata da un cospicuo numero di manoscritti e discussa in Lucarini, "De Hermiae", 80-83, rimane invece aperta: come lo stesso L. ammette (pp. xxx-xxxi e nota nr. 97), Siebenkees mai visitò le biblioteche fiorentine e comunque questi scolî possono essere frutto della confusione delle carte da lui lasciate alla sua morte; in ogni caso, rimane la necessità di un supplemento di indagine, visto che nessuno degli scolî incriminati ricade nei saggi di collazione disponibili ai nostri editori. ${ }^{30}$ In questo quadro risulta però poco perspicuo, a mio avviso, il ruolo avuto dal Ven. Marc. gr. 228(V), da cui pare derivi l'unico estratto pubblicato da 2007.

${ }^{24}$ Per tutto ciò, mi sia lecito rimandare a D. Cufalo, Scholia Graeca in Platonem, I, Roma

${ }^{25}$ I. Ph. Siebenkees, Anecdota Graeca, a cura di I. A. Goez, Norimbergae 1798. Mi è caduto l'occhio su un errore di stampa nelle ultime due righe di pag. lvi, dove si legge $\mu \alpha v \tau \varepsilon i ́ o v$,

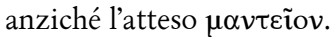

${ }^{26}$ Si tratta dell'estratto relativo a Phaedr. 227a, corrispondente a Herm. 19.9-12, rièdito da

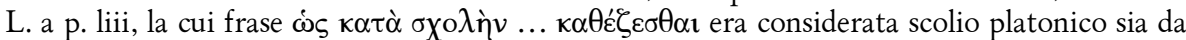
C. F. Hermann, Platonis Dialogi secundum Thrasylli tetralogias dispositi, Lipsiae 1858, VI, 262, sia da W. C. Greene, Scholia platonica, Haverfordiae 1938, 68: si veda ora Cufalo, Scholia Graeca, 117-118, con la relativa nota d'apparato.

${ }^{27}$ Si ricordi che il volume di Siebenkees, morto nel 1796, fu pubblicato postumo, dunque l'opera mancò di una summa manus da parte dell'autore.

${ }^{28}$ Si tratta delle biblioteche dai cui manoscritti Siebenkees dedusse gli scolî platonici. Traiamo l'informazione dalla stessa prefazione, riprodotta da L. alla nota 93 p. xxx. In fondo a questa stessa nota, si trova un piccolo refuso: «adhuibuisse» per «adhibuisse».

${ }^{29}$ Il codice è ormai agevolmente consultabile all'indirizzo http://gallica.bnf.fr/ark:/12148/ btv1b10507219n.

${ }^{30}$ I saggi di collazione dei nostri editori ricoprono le seguenti quattro sezioni: 1.1-17.15 (1.1-16.12 C.), 52.1-70.26 (48.12-66.15 C.), 107.17-115.27 (102.1-110.21 C.), 231.23-236.18 (221.6225.20 C.). 
Siebenkees come proveniente da Hermias e riprodotto da L. alla nota 134 p. $\mathrm{lv}$, codice che certo non fa parte della famiglia $\rho$ e che è privo del terzo libro, dal quale provengono molti estratti èditi dallo studioso tedesco. ${ }^{31}$

Viene quindi dedicato un breve spazio ad un ampio scolio di mano di Niceforo Gregoras contenuto nel f. 225v del celeberrimo Bodl. Clark. 39, il codice $\mathrm{B}$ di Platone. Esso però non risulta utile per la constitutio textus, in quanto proveniente direttamente dal testo di Hermias, probabilmente proprio dal codice Par. gr. 1810, come già dimostrato da Vezio Nibetti. ${ }^{32}$

Il breve capitolo successivo è dedicato ad un opuscolo di Michele Psello,

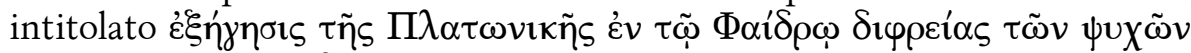

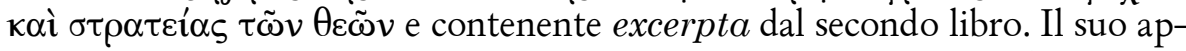
porto è molto ridotto, ma ci permette di recuperare almeno un tó a 142.30.

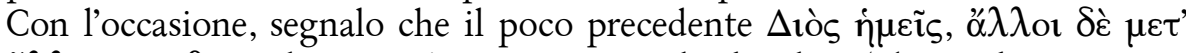

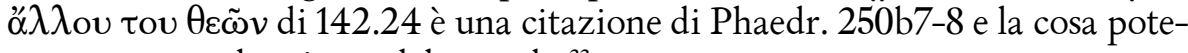
va essere segnalata in qualche modo. ${ }^{33}$

Gli altri capitoli curati da L. sono: De locis aliorum scriptorum ab Hermias allatis (pp. xl-xli), in cui si precisa il metodo seguito nel pubblicare le citazioni da altri autori nei casi di presunte o sicure corruzioni; De Platonis exemplari ad Hermia adhibito (pp. xli-xlv), in cui si sostiene una maggiore consonanza del testo di Hermias con quello dei codici Marc. gr. App. Cl. IV.I (T) e Vindob. Suppl. gr. $7(\mathrm{~W})$ di Platone; ${ }^{34}$ De Hermiae editionibus (pp. xlv-xlviii); Stemma (pp. xlviii-xlix), in cui viene anche proposto a confronto, sebbene a mio avviso in modo un po' indelicato, lo stemma di Couvreur; Haec editio (pp xlix-li).

Molto più importante è il capitolo De librorum necessitudinibus (pp. xxxiii-xxxix), scritto, come già accennato, a quattro mani, in cui, assolvendo a quanto promesso in L., "De Hermiae", 87, gli autori si concentrano soprattutto sui codici più antichi, l'Ambr. D 166 inf. (c), il Laur. Conv. Soppr. 103 (L, datato al 1358), il Laur. Conv. Soppr. $78(\mathrm{l})$ e il Ven. Marc. gr. 228(V).

Come è noto, Luna e Segonds, studiando la tradizione manoscritta dell'In Parmenidem di Proclo, avevano sostenuto che il Parigino, dopo essere stato trascritto e corretto da Giorgio Pachymeres $\left(\mathrm{A} \mathrm{e}^{1}\right)$, fu ricopiato da clV e quindi, in seguito a danneggiamenti a causa dell'umidità, restaurato da ben

${ }^{31}$ Per la dimostrazione della dipendenza da V, si veda Lucarini, "De Hermiae”, 87.

${ }^{32}$ V. Nibetti, "Un codice di Proclo ed Ermia alessandrino tra Giorgio Pachimere e Niceforo Gregora (Parisinus Graecus 1810)”, RFIC 136, 2008, 385-395; cf. anche Cufalo, Scholia Graeca, 122 (in apparato agli scolî 30-36 e 38-46).

${ }^{33}$ Che fosse una citazione sfuggì anche a Couvreur, ma non a Bernard, Hermeias von Alexandrien, 259 n. 194.

${ }^{34}$ Alla nota 114 pag. xli meritava di essere citato anche A. Carlini, "I lemmi del Commento di Proclo all'Alcibiade I e il codice W di Platone”, SCO 10, 1961, 179-187, se non altro perché antesignano nel campo. 
tre mani $\left(\mathrm{A}^{2}, \mathrm{~A}^{3}\right.$ e $\left.\mathrm{A}^{4}\right)$ : le prime due, $\mathrm{A}^{2}$ e $\mathrm{A}^{3}$, non sono molto anteriori al 1358, anno in cui fu trascritto $\mathrm{L}$, che infatti conosce tutte quelle correzioni, mentre la terza risale all'inizio del Cinquecento.

Questa tesi, almeno per quanto riguarda Hermias, viene esclusa da L.-M. ${ }^{35}$ secondo i quali non ci sono argomenti sufficienti per sostenere che L sia in qualche modo più recente rispetto a clV. La conseguenza più importante di questa conclusione è che i nostri editori rinunciano ad operare una esatta distinzione fra le mani e, recependo la simbologia di Couvreur, indicano con la sigla $\mathrm{A}^{a}$ le lezioni di $\mathrm{A}$ «ubi prima manus dispici nequit» e, con la sigla a, $\mathrm{i}$ casi «ubi prima manus dispicitur».

Ho sempre trovato molto poco intuitiva questa nomenclatura e, nonostante la lunga confidenza con l'edizione di Couvreur, ancora oggi mi ritrovo spesso costretto a consultare il conspectus siglorum, per ricordare il significato di queste sigle. Tuttavia, il vero problema è un altro. Si può discutere sulla bontà della distinzione fra le mani che Luna e Segonds chiamano $\mathrm{A}^{2} \mathrm{e}$ $\mathrm{A}^{3}$, si può riconoscere che le differenze sono veramente molto sottili e, di fatto, limitate al colore dell'inchiostro, più scuro nell'ultima, tuttavia il rischio insito nell'uso di queste sigle generalizzanti è sempre quello di obliterare informazioni che magari uno studioso del futuro avrebbe potuto valorizzare.

Non è certo possibile in questa sede procedere a nuove collazioni, che comunque c'è da ritenere che i nostri studiosi abbiano effettuato con la loro consueta massima cura, tuttavia, a mio avviso, la mancata distinzione delle mani correttrici di A rende alquanto difficile verificare il seguito delle argomentazioni. Infatti, dopo avere sostenuto, sulla base dei casi in cui cLlV hanno un testo migliore di quello di $\mathrm{A}^{\mathrm{a}}$, che essi derivano tutti da $\mathrm{A}$ prima che quest'ultimo abbia subito i danni e dunque i successivi restauri, L.-M. si trovano costretti, visti i casi contrari in cui $\mathrm{A}^{\mathrm{a}}$ ha un testo migliore rispetto a quello degli apografi, a postulare un intermediario $\alpha$, dal quale cLIV deriverebbero, indipendentemente l'uno dall'altro. ${ }^{36} \mathrm{~A}$ favore dell'esistenza di $\alpha$, ricopiato da A anteriormente all'intervento dei restauratori, gli autori adducono altresì il fatto che gli errori degli apografi si osservano tutti nei luoghi in cui A stesso si presenta danneggiato, poiché, sostengono, «quomodo enim cogitari potest, $\alpha$ fere solis illis locis dormitavisse, quos postea mador in A laesit?».

Questa tesi è verosimile, ma a mio avviso non è l'unica possibile. Essa si fonda sul postulato secondo cui A, come uscito dalla penna di Pachymeres, fosse del tutto esente da errori, come se il nostro seppur dotto copista non potesse commettere errori di trascrizione, come tutti noi. Inoltre, trascura la possibilità che i correttori di $\mathrm{A}$, anch'essi uomini dotti, non si siano limitati a cercare di ricostruire il testo, per così dire, "originale", ma abbiano fatto ri-

${ }^{35}$ In realtà, già da C. Moreschini, "Ermia alessandrino nel Medioevo e nel Rinascimento", SCO 55, 2009, 283-300, in particolare 295-296.

${ }^{36}$ Segnalo un piccolo refuso a p. xxxvii riga 1: «exempar», anziché «exemplar». 
corso, qua e là, alla divinatio, con risultati talora buoni, talaltra meno buoni. Poi, non considera che, come gli interventi dei correttori, così il deterioramento dovuto all'umidità potrebbe non essere intervenuto, per così dire, da un giorno all'altro, ma essersi manifestato e aggravato in un arco di tempo più lungo. Infine, non dobbiamo nemmeno dimenticare che tutti i cinque codici, A da una parte e cLlV dall'altra, sono stati trascritti in un arco di tempo di circa cinquanta/sessanta anni e probabilmente tutti in uno stesso ambiente, al punto che non si può nemmeno escludere che vi siano stati fenomeni di contaminazione fra di loro, anche se questa eventualità viene cautamente esclusa dai nostri autori (p. xxxix e n. 110).

Di queste medesime considerazioni anche L.-M. si mostrano pienamente consapevoli. Si noti infatti che, partendo dal caso in cui in cui è palese che c dipende direttamente da $\mathrm{A},{ }^{37}$ osservano che "viros doctos, quos non fallebat pretium codicis Parisini olim illius Pachymeris, non solum $\alpha$, ubi omnia sine ullo labore legere poterant, sed etiam A hic illic adhibuisse» (p. xxxvii). Inoltre, subito dopo, sostengono che, poiché non di rado i recenziori hanno una lezione buona contro i correttori di A, $\alpha$ dovette avere davanti a sé un codice già danneggiato, ma ancora meglio leggibile (p. xxxviii). Infine, a p. xxxix, ammettono, con Luna e Segonds, che almeno il copista di 1 «et de Hermia aliquid boni excogitasse fere certum est».

In conclusione, mi pare che la tesi di L.-M., secondo i quali cLlV dipendono direttamente da un intermediario $\alpha$, ricopiato da A quando esso era già in parte danneggiato ma non ancora restaurato, tuttavia utilizzarono saltuariamente anche A stesso, sia certo plausibile, ma necessiti di ulteriori verifiche, che non possono prescindere dall'individuazione e dalla distinzione delle mani dei correttori.

Queste considerazioni, pur importanti per la storia del testo, poco incidono in definitiva sulla constitutio textus, e, in ogni caso, L.-M., partendo dalla pur ottima base costituita dall'edizione di Couvreur, hanno rivisto con massima cura $\mathrm{A}$, non di rado correggendo le letture del loro predecessore, ${ }^{38} \mathrm{e}$ hanno prodotto una pregevolissima edizione, che si avvale altresì delle lezioni più interessanti degli apografi cLIV e, ove disponibile, della tradizione indiretta.

L'apparato, in genere corposo, è distribuito su due livelli: quello superiore registra fonti e loci similes, in gran parte fondato, come dichiarano gli stessi autori (p. li), sugli apparati di Couvreur e di Bernard; quello inferiore è dedicato a varianti e congetture. Note marginali segnalano sia la paginazione di Couvreur sia, seguendo il buon esempio di quest'ultimo, la foliotazione del codice A.

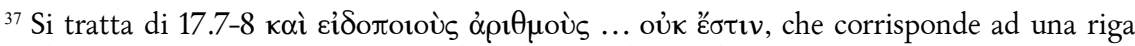
esatta di A. La cosa era già stata segnalata da M. Schanz, "Zu Hermeias", Hermes 18, 1883, 129-136.

${ }^{38}$ Alcuni errori di Couvreur sono elencati alla nota 129 pp. xlix-l. 
Il testo proposto è caratterizzato da tantissime congetture, alcune accolte, altre solamente suggerite in apparato, nel quale peraltro i curatori si soffermano molto spesso a giustificare le loro scelte o a discutere le proposte altrui con annotazioni brevi, ma efficaci. ${ }^{39}$

Non è possibile, né avrebbe tanto senso, discutere qui tutte le tantissime scelte testuali che i curatori hanno proposto nelle quasi trecento pagine della loro edizione. Mi limiterò pertanto a pochi saggi, in parte selezionati a partire da un paio di lavori preparatorî di L. ${ }^{40}$

Iniziamo la rassegna con una osservazione, per cosi dire, "personale", re-

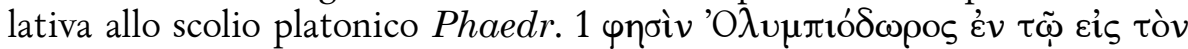

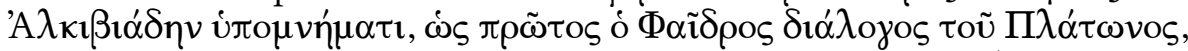
tramandato in forma pressoché identica in margine ad Hermias (è riprodotto da L.-M. in apparato a 13.7) e nel codice W di Platone (Vind. Phil. gr. 7, saec. XI ex.) e, con lievi differenze, in T (Marc. App. Cl. IV 1, manu <Ephraem monaci>, saec. X me.). ${ }^{41}$ Nella mia edizione avevo sostenuto che la versione di W fosse quella, per cosi dire, "originale" e che lo scolio derivasse da Hermias, con ciò intendendo non che Hermias l'avesse desunta da Olimpiodoro, cosa per ovvi motivi impossibile, ma che lo scoliaste avesse trovato la nota in un codice di Hermias già fornito di questo scolio. ${ }^{42}$ L'ipotesi è resa molto verosimile dal fatto che l'archetipo del nostro autore era un codice della cosiddetta "Collezione Filosofica", ovvero di quel gruppo di manoscritti, individuato da Thomas W. Allen, cui appartengono sicuramente, tra gli altri, il celeberrimo codice A di Platone (Paris. gr. 1807, saec. IX po. me.), antigrafo del summenzionato T, e il più importante codice di Olimpiodoro (M: Marc. gr. 196, saec. IX po. me.). ${ }^{43}$

${ }^{39}$ Una manciata di esempi già a pagina 1, in relazione alle righe 12, 18 e 20.

${ }^{40}$ C. M. Lucarini, "Per il testo di Ermia Neoplatonico", Philologus 152.1, 2008, 148-153; id., "Per il testo di Ermia Neoplatonico (II)", MD 69, 2012, 239-257.

${ }^{41}$ Cufalo, Scholia Graeca, 117.

${ }^{42}$ Per la mia definizione di "fonte", si veda Cufalo, Scholia Graeca, xxvii. Si noti che anche Couvreur, in apparato, scriveva, in relazione allo scolio, "quod non Hermiae tribuendum» (p. 13).

${ }^{43}$ Cf. T. W. Allen, "Palaeographica III. A Group of Ninth-Century Greek Manuscripts", $J P h$ 21, 1893, 48-55. Per la bibliografia più moderna, mi limito a rimandare a L. Perria, "Scrittura e ornamentazione nei codici della "collezione filosofica»", $R S B N 28,1991,45-111$. Di recente, Filippo Ronconi ha ridimensionato il ruolo e la consistenza di questa "Collezione", ma, in definitiva, nemmeno lo studioso nega l'unitarietà del gruppo platonico, quello che a noi interessa. Si vedano, in proposito: F. Ronconi, "Le silence des livres. Manuscrits philosophiques et circulation des idées à l'époque byzantine moyenne”, in L. Del Corso e P. Pecere (eds.), Il libro filosofico dall'antichità al XX secolo, Quaestio 11, 2011, 169-207; Id., "La collection brisée. Pour une étude des milieux socioculturels liés à la "collection philosophique»", in P. Odorico (ed.), La face cachée de la littérature byzantine. Le texte en tant que message immédiat, Actes du colloque international (Paris, 5-7 juin 2008), Dossiers Byzantins 11, Paris 2012, $137-$ 166; Id., "La collection philosophique: un fantôme historique", Scriptorium 67, 2013, 119-140. Anzi, in quest'ultimo articolo lo studioso esalta il «niveau qualitatif du groupe $B$, qui s'avère être - dans le cadre culturel méso-byzantin - un véritable trésor au contenu rarissime» (p. 134). 
Oggi non sarei più così sicuro della bontà di questa tesi. La compresenza di più codici in un medesimo ambiente, quello della "Collezione Filosofica", potrebbe indurci ad individuarne la fonte proprio nel commento di Olimpiodoro, dal quale questa nota sarebbe stata estrapolata e quindi ricopiata in margine sia ai manoscritti platonici sia a quello di Hermias. Meno probabile l'ipotesi alternativa che esso sarebbe stato aggiunto in margine ad Hermias da Pachymeres, ${ }^{44}$ se non altro perché questi sembra molto poco interessato all'apparato scoliastico a Platone, se è vero che, dalla sua penna, con inchiostro rosso, sono stati trascritti solo questo, al f. 20r, e il nr. 42 (dove scrive -

Del resto, già Couvreur aveva notato che lo scolio «non Hermiae tribuendum» (p. 13 in app.) ed in effetti, a ben guardare, esso è stato trascritto accanto alle prime righe del Fedro, subito accanto alla sigla che indica l'interlocutore, in questo caso Socrate, nel piccolo spazio fra il testo platonico e quello di Hermias. Non vi può essere dubbio sul fatto che Pachymeres abbia strutturato la pagina in modo da potersi lasciare lo spazio per trascrivere lo scolio,

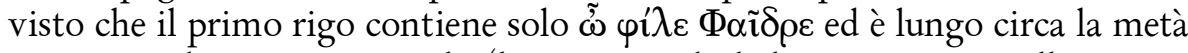
rispetto ai due successivi righi (la porzione di dialogo trascritta nella pagina

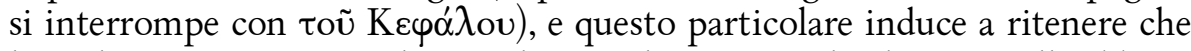
lo scolio sia strettamente legato al testo platonico, e che dunque nulla abbia a che vedere con il nostro commentario. Insomma, Pachymeres ha ricopiato il testo platonico, omettendo tutti gli scolî che trovava nella sua fonte, eccezion fatta per questo e per il nr. 42: a lui interessava il commentario di Hermias più che gli sparsi scolî, ma rimarrà sempre un mistero perché abbia mantenuto i due suddetti.

En passant, si può notare che sia lo scolio, sia le indicazioni dell'interlocutore, sia i numerali che collegano il testo platonico con le relative note di Hermias, sia le altre ornamentazioni, sono tutte scritte con il medesimo inchiostro rosso.

Nel rapportarsi a queste rubriche, i nostri editori seguono il comporta-

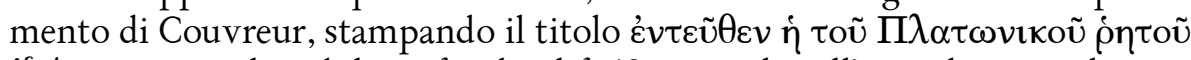

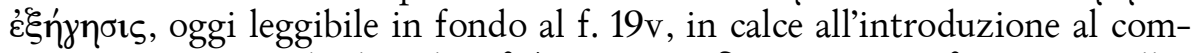

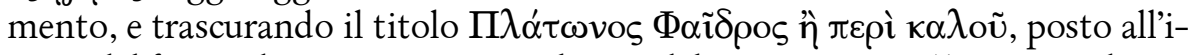
nizio del f. 20r, direttamente sopra il testo del commentario, ${ }^{46}$ ma se ne disco-

\footnotetext{
${ }^{44}$ In tal caso, è molto più probabile che Pachymeres abbia desunto la nota da qualche manoscritto platonico, e non da Olimpiodoro: quest'ultimo è oggi tramandato dal Marc. gr. 196 e da una serie di apografi, tutti ben posteriori a Pachymeres.

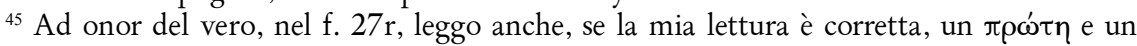
$\delta \varepsilon v ́ \tau \varepsilon \rho \alpha$, che forse sostituiscono, rispettivamente, gli scolî nrr. 43 e 44 .

${ }^{46}$ Poco perspicua l'indicazione di apparato dei nostri editori (p. 14), dai quali non risulta chiaro che le due rubriche sono collocate in fogli diversi e opposti. Poco chiara è anche l'indicazione «in A margine» riferita allo scolio platonico, che, di fatto, è collocato sì in margine al Fedro, ma al centro della pagina.
} 
stano quando non accolgono le integrazioni dei lemmi, la prima delle quali è $\tilde{\omega} \varphi \lambda^{\prime}{ }_{\varepsilon} \Phi \alpha \tilde{i} \delta \rho \varepsilon$ posto dopo il numero $\alpha^{\prime}$ (14.8 L.-M. = 13.5 C.).

Quest'ultima scelta è senza dubbio ineccepibile, ma forse sarebbe il caso di riconsiderare in generale il ruolo e il significato di tutti questi elementi accessori presenti in A, che potrebbero anche essere frutto della cura edi-

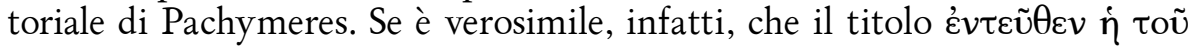

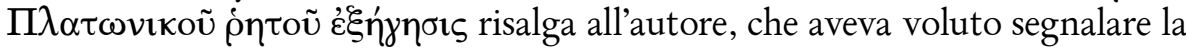
fine della prefazione e l'inizio del vero e proprio commentario, meno sicura è la cosa per i numerali, il cui ruolo è collegare il testo del commento a quello platonico. Inoltre, mi sarei aspettato alcune indicazioni sugli scolî figurati, anch'essi rubricati, leggibili sui margini inferiori dei ff. 45v, 46r e 77r, di cui in questa edizione non è rimasta traccia, sebbene fossero ancora documentati da Couvreur alle pag. 103, 105 e 221 della sua edizione.

Si lamenta una certa lacuna di indicazioni, pur a fronte di una ampia nota d'apparato, anche a 2.8-16, dove, nel contesto di un riassunto del dialogo (la

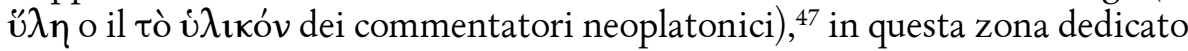
a Phaedr. 234-235, vengono inserite delle righe che invece riassumono Phaedr. $263 \mathrm{~d}-264 \mathrm{c}$, a loro volta commentate specificatamente a partire, grosso modo, da 239.13. Che ci fosse qualcosa di anomalo non era sfuggito a Cou-

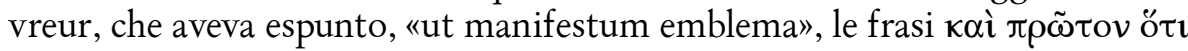

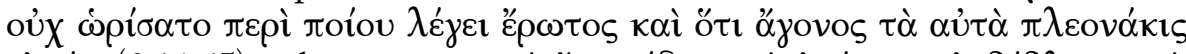

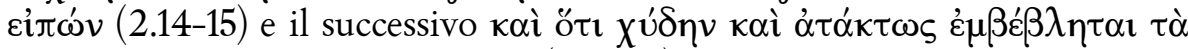

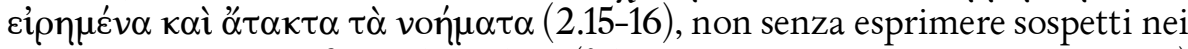

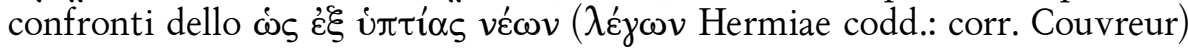
che si trova in mezzo alle due frasi incriminate.

C'è da ammettere che il flusso del discorso di Hermias è un po' confuso. A 2.5-6 aveva appena affermato, sulla scorta di Phaedr. 234e7-8, che lo scritto-

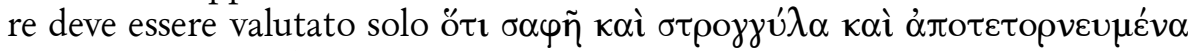

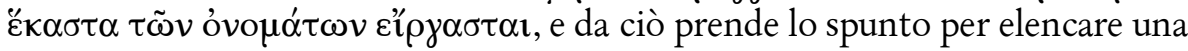
serie di motivi di critica verso il discorso di Lisia, attraverso una sequenza di

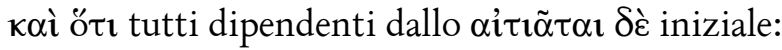

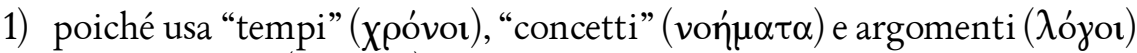
in modo disordinato ("丷 $\tau \alpha \kappa \tau \alpha)$, precisando che:

a) ha cominciato dalla fine ( $\dot{\alpha} \pi$ ò $\tau \varepsilon \lambda \varepsilon v \tau \tilde{\eta} \varsigma ~ \eta ้ \rho \xi \alpha \tau o)$, ovvero dalla fase successiva all'innamoramento, anziché dall'inizio di esso;

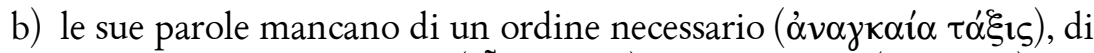

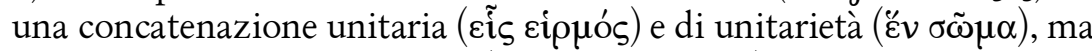

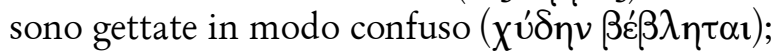

${ }^{47}$ Sulle sezioni in cui si era suddivisa l'introduzione, si legga ad esempio Prol. in Plat. Philosoph. 16-17, in particolare 16.7-22 sulla ü $\lambda \eta$. 


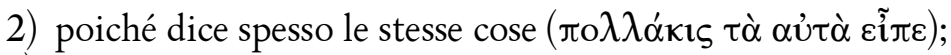

3) poiché non ha definito sin dall'inizio di quale amore sta parlando;

4) poiché, in quanto †öyovosł, dice più di una volta le stesse cose ( $\tau \grave{\alpha}$

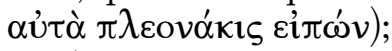

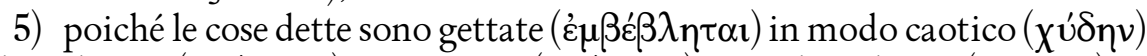

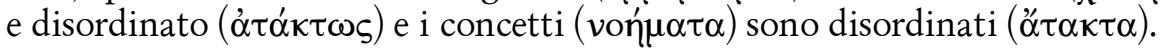

Come si può notare, i punti $1 \mathrm{e} 1 \mathrm{~b}$ corrispondono al punto 5 per l'uso dei

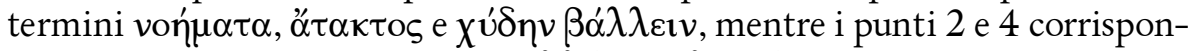

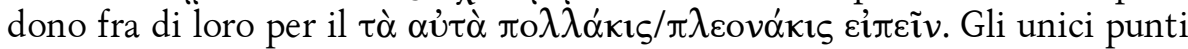
"originali" rimangono pertanto il $1 \mathrm{a}$ e il 3 , ma anche il $\mathrm{lb}$ per la parte relativa

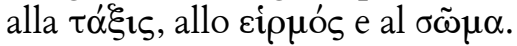

Se, come si è detto, Couvreur aveva cassato i punti 3,4 e 5 , i nostri editori si mostrano più indulgenti, salvando almeno il punto 5 .

Il problema è ben più complesso. In apparato, L.-M. spiegano che in tutta questa sezione (2.8-16) sarebbero confluiti due scolî, definiti "ambo aliena», il primo da $\alpha \mathbf{i} \tau \mathbf{\imath} \tilde{\tau} \alpha \boldsymbol{\imath} \delta \grave{\varepsilon}(2.8)$ a $\varepsilon \tilde{i} \pi \varepsilon(2.14)$, e dunque abbracciante i punti 1

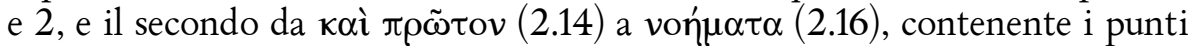
$3-5$, che a sua volta costituirebbe una "prioris iteratio". Che però il "secondo scolio" sia una "prioris iteratio" non è così pacifico, visto che in esso abbiamo il punto 3 , che anzi risulta, come abbiam testé visto, "originale" ed è motivato da Phaedr. 263d7-e1.

D'altra parte, ciascuno dei punti trova riscontro nel testo platonico: il 1a

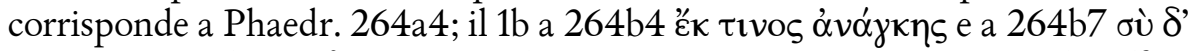
हैX

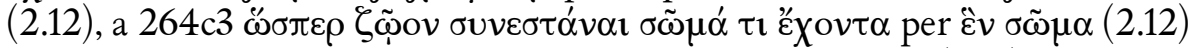

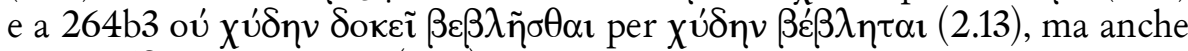
per il $\chi \cup ́ \delta \eta v$ del punto 5 (2.16). Solamente i punti 2 e 4 , come correttamente notato in apparato dai nostri, trovano paralleli nella sezione corrispondente del Fedro, ovvero 235a4-6 e a7-8.

In un simile contesto di riferimenti incrociati, è lecito chiedersi per quali motivi la scure dei nostri editori, ancorché meno tagliente di quella di Couvreur, sia caduta solo sul punto 4 , che peraltro è l'unico a presentare un argomento "nuovo". Mi chiedo in altri termini come mai queste presunte inserzioni, se è vero che sono "aliena», siano state sostanzialmente accolte nel testo dai nostri editori. La loro scelta può anche essere condivisibile, ma, stante la (ottima) abitudine di produrre ampie note d'apparato, mi chiedo se non fosse possibile fornire al lettore maggiori ragguagli su di essa, che magari chiarissero per quale motivo e da chi queste righe siano state inserite in questo punto del commentario.

Possiamo sospettare che gli editori siano stati spinti a cassare questa sola sezione dalla ripetizione del precedente punto $2, \mathrm{ma}$, in questo caso, ci si può chiedere come mai abbiano mantenuto la parte successiva, espunta da Cou- 
vreur. In ogni caso, l'assetto testuale di L.-M. non risulta nemmeno del tutto

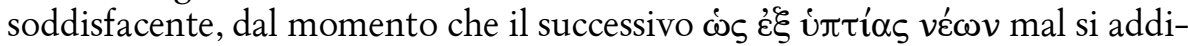
ce al $\tau \grave{\alpha} \alpha \hat{\tau} \tau \grave{\alpha} \varepsilon \tilde{i} \pi \varepsilon$ e infatti, non a caso, in apparato gli editori suggeriscono di trasporlo dopo $\eta^{\prime} \rho \xi \alpha \tau$ o del r. 9. ${ }^{48} \grave{\mathrm{E}}$ invece da escludere che questa espunzione sia anche legata al sospetto ózovos, che però non è emendabile, se non altro perché ricompare identico, e in un contesto del tutto simile, a 3.10. ${ }^{49}$

In conclusione, è vero che sussiste in questo passo una certa incoerenza espositiva, ma, a mio avviso, questa incoerenza, tra l'altro ripetuta poco dopo, a 3.10-12, che interrompe una esposizione relativa a Phaedr. 236a, andrebbe spiegata in altri modi, non attraverso espunzioni totali o parziali, che tra l'altro, come si è visto, non riescono nemmeno a risultare del tutto soddisfacenti.

Proseguendo con la carrellata, arriviamo a 30.5-6, dove gli editori stampa-

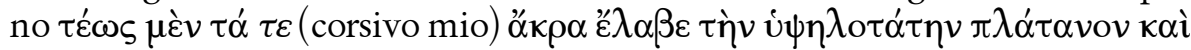

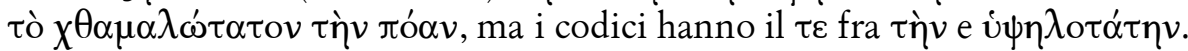
A me pare che il testo di L.-M. faccia perdere la contrapposizione fra il platano e l'erba. L'autore sta osservando che, fino ad ora ( $\tau$ '́c $\left.\varsigma_{\varsigma}\right)$, Platone aveva preso in considerazione i due estremi ( $\tau \grave{\alpha} \alpha$ $\alpha \rho \alpha)$ e quindi precisa che essi sono sia il platano, che è altissimo, sia l'erba, che invece è a terra; successivamente, prenderà in considerazione anche ciò che è in mezzo, ovvero tò ớzvos.

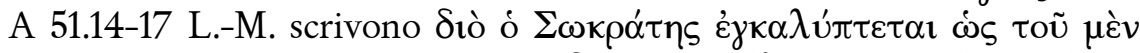

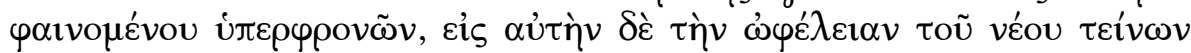

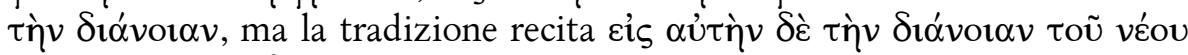

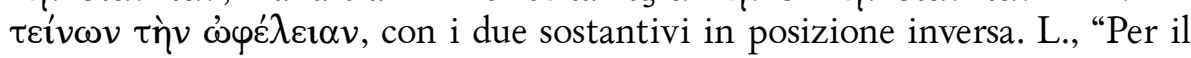

${ }^{48}$ Qualche perplessità nutro anche verso la congettura di Couvreur vécov, per il tràdito

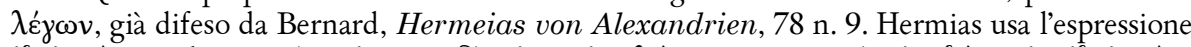

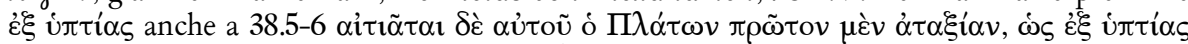

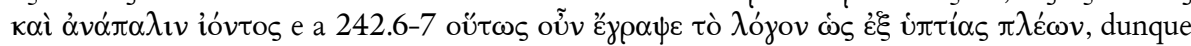
sempre in contesti identici in cui si critica il "disordine" del discorso di Lisia. Il verbo recuperato

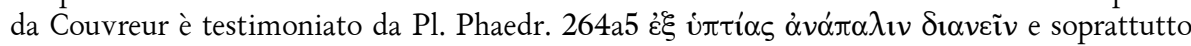

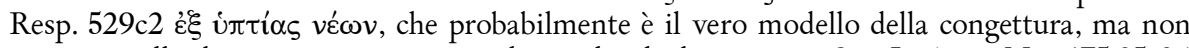
compare nelle altre tre attestazioni neoplatoniche che ho reperito: Syr. In Arist. Met. 175.25-26

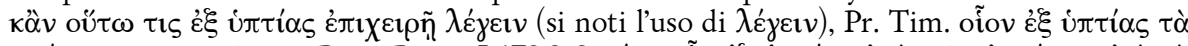

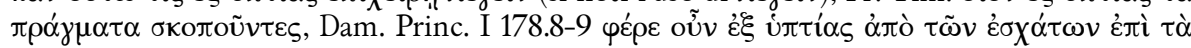

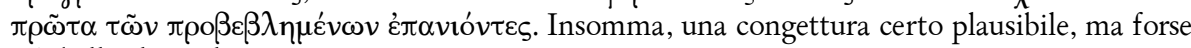
più bella che realmente necessaria.

${ }^{49} \mathrm{La}$ migliore correzione possibile è a mio avviso ö $\tau \varepsilon X v o s$, già suggerito da L. in apparato a 3.10 sulla base del confronto con 10.16, cui possiamo aggiungere ad esempio 239.9-14. Nemmeno è da escludere áyevvĩs, sempre suggerito da L. e confortato da Pl. Phaedr. 264b6, ma purtroppo il duplice errore non consente di intervenire in questi passi. Come però mi segnala l'amico Vincenzo Muggittu, che ringrazio, il termine äyovo s può qui avere il senso di "sterile", ad indicare un discorso "che invece di partorire un esito va a ritroso" (parole sue). In tal senso si

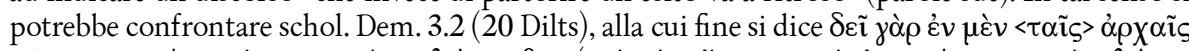

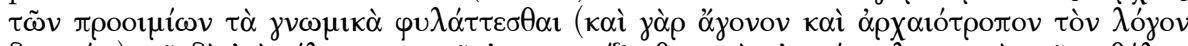

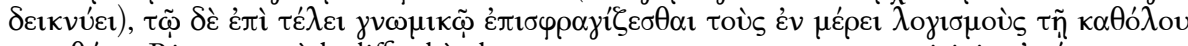
$\pi \rho \circ \theta \eta \dot{k}$. . Rimane però la difficoltà che questa sequenza presenta un participio eì $\pi \omega^{\prime} v$, mentre in tutti gli altri argomenti si ha l'indicativo. 
testo", 151, ha osservato che "la Sióvor $\alpha$ non è mai trattata da Ermia come un qualcosa che possa essere aiutato o danneggiato", mentre in lui sarebbe «abbastanza comune l'idea per cui il compito di Socrate sia quello di giovare ai giovani in generale e a Fedro in particolare». Agli esempi addotti da L., si può

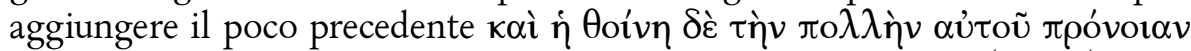

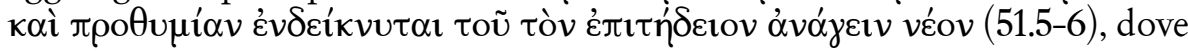
la $\pi$ óvor $\alpha$ viene appunto riferita a Socrate, ${ }^{50}$ tuttavia la formulazione tràdita non è in sé impossibile e gode anch'essa di paralleli, seppur esterni al no-

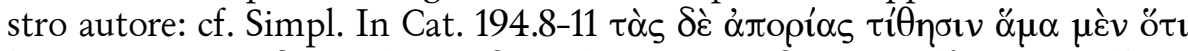

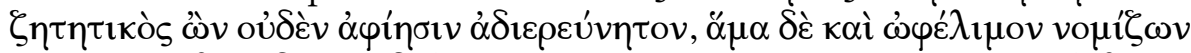

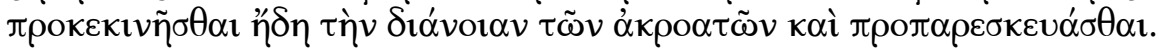

A 55.7, L. segnala in apparato la sua proposta di integrare un $\pi \tilde{\alpha} \varsigma$ dopo $\dot{\varepsilon} \pi \imath \theta u \mu \tilde{\omega} v$. L'integrazione non è necessaria: dopo aver detto che la $\varphi \imath \lambda{ }^{\prime} \alpha$ rientra nel ý́vos della émı $\theta u \mu i \alpha$, ora si precisa che non è vero il contrario (oủk

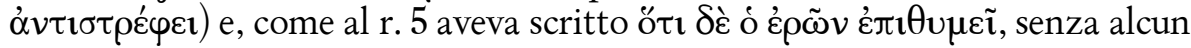

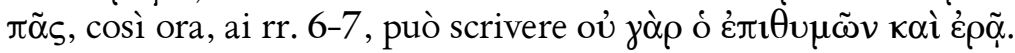

Piuttosto controverso è il passo di 91.3-17, nel quale si parla delle capacità profetiche delle statue. Un primo problema affligge già le prime righe, in cui si spiega che la telestica, attraverso una serie di rituali, "vivifica" le statue e le rende in grado di ricevere una illuminazione dal dio. Il testo reci-

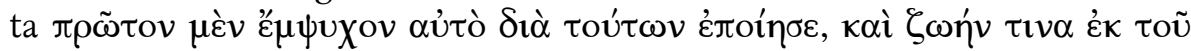

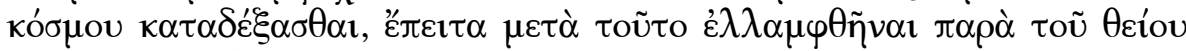

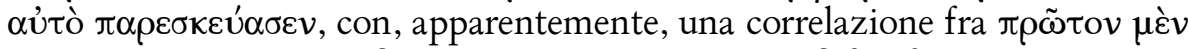

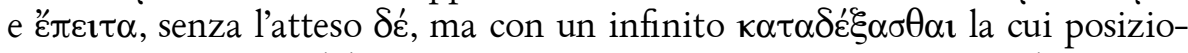
ne sintattica non è del tutto perspicua. Couvreur aveva proposto di integra-

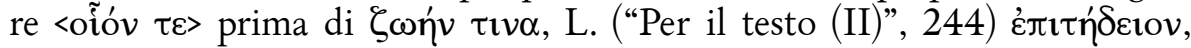
ma nessuna di queste due integrazioni è entrata nel testo, giacché gli editori hanno preferito accogliere il suggerimento dell'anonimo referee della rivista,

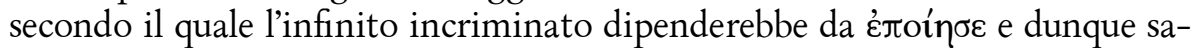

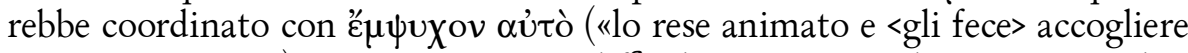
una certa vita...»). Provo una certa difficoltà a comprendere questa scelta, ma sarei anche curioso di sapere quale sia stata la reale proposta del referee, visto che nel succitato articolo (alla nota 2 ), a differenza che nella nota di

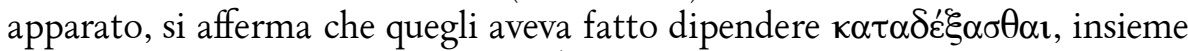
a $\dot{\lambda} \lambda \lambda \alpha \mu \varphi \theta \tilde{\eta} v \alpha \mathbf{l}$, da $\pi \alpha \rho \varepsilon \sigma \kappa \varepsilon v ́ \alpha \sigma \varepsilon v$ ( «lo rese animato e lo apparecchiò a ricevere..., quindi, dopo ciò, ad essere illuminato...»), tra l'altro con una struttura

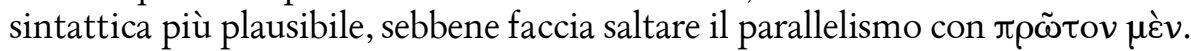

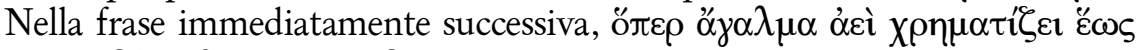

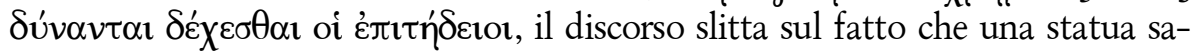

${ }^{50}$ Un altro parallelo può essere istituito con Ol. In Alc. 14.10-19, dove, facendo riferimento

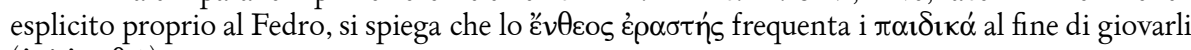

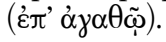

ExClass 21, 2017, 227-242 
rebbe in grado di vaticinare sempre, finché i preposti sono in grado di riceverne i vaticinî. Lucarini ("Per il testo (II)", 245) aveva sospettato questa frase, pro-

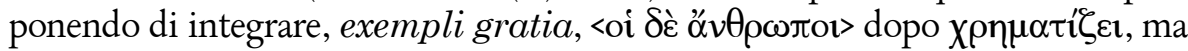
di questa integrazione nella nostra edizione non rimane traccia alcuna, se non una laconica nota di apparato "post h. v. lac. indic. Lucarini ${ }^{4}$ ». In effetti, appare pacifico, come spiegato nell'articolo, che gli غ̇ंı

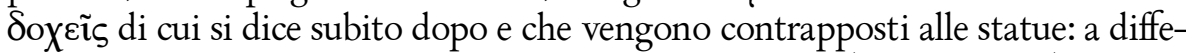
renza di queste ultime, che "hanno una attività costante ( $\mu \varepsilon \dot{v} \varepsilon \mathbf{\varepsilon} \varepsilon \varphi \varepsilon \xi \tilde{\eta} \varsigma)$ ", finché la "carica magica" non si esaurisce e si deve quindi procedere ad un nuovo rituale vivificatorio, l'attività dei Teurgi alterna fasi di operatività e di riposo, perché l'anima umana non può reggere ininterrottamente l'interazione col divino. La scelta di mantenere il testo tràdito, pertanto, si rivela a mio avviso felice e, al massimo, si lamenta una certa laconicità nella nota d'apparato.

A 99.21-22, L., "Per il testo (II)", 246, sostiene che l'ampia espunzione di

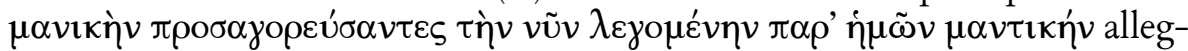
gerirebbe l'argomentazione, eliminando l'anticipazione di un concetto subito dopo più diffusamente spiegato ed evitando che il successivo ö $\pi \varepsilon \rho$ ǒvo $\mu \alpha$ sembri riferito a $\mu \alpha v \iota \kappa \eta ̣ v$, anziché a $\mu \alpha v i \alpha$.

La tesi è plausibile, ma non si può nemmeno escludere che proprio la

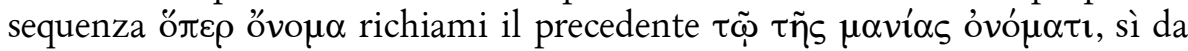
rendere meno equivoca l'espressione. Per quanto riguarda invece la duplicazione, ci sarebbe da chiedersi perché un copista avrebbe dovuto interpolare una sequenza così lunga, visto che in definitiva la spiegazione risulta comunque comprensibilissima.

Pertanto, credo che possa essere riabilitata la proposta di Couvreur, ov-

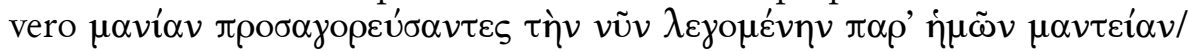

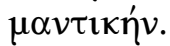

Un'altra trasposizione viene proposta a 209.11-12, dove, di contro al tràd-

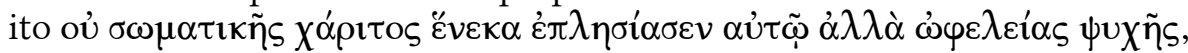

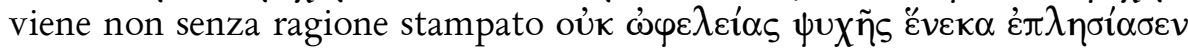

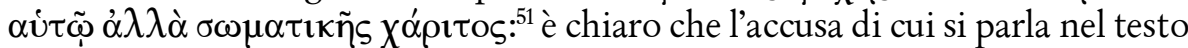
ha senso se rivolta ad un amante che approccia l'amato per il piacere fisico, anziché per giovare alla sua anima. Mi chiedo però se non sarebbe stato più

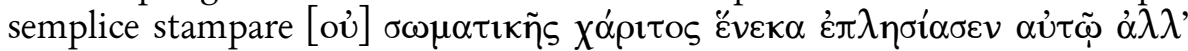

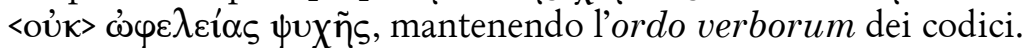

Vero è che la sequenza proposta dagli editori è più frequente in Hermias e ritorna poche righe sotto (209.17-18; anche, ad esempio, a 238.4-5), ma possiamo confrontare 2.10-11, in cui, spiegando una delle critiche rivolte avverso

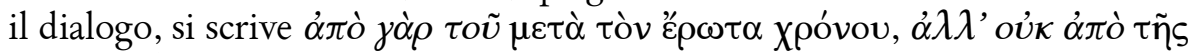

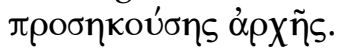

${ }^{51}$ In apparato, in relazione alla riga 12 , le citazioni testuali, sia quella del testo stampato sia quella della versione tràdita, sono abbreviate, ma, in entrambi i casi, si scrive $\alpha$. anziché $\alpha$. 
Concludendo questa fin troppo lunga carrellata, bisogna però ammettere che è facile trovare da ridire su qualcuna delle innumerevoli scelte testuali dei nostri editori. Ciò è facile in qualunque edizione, soprattutto se di un testo molto lungo, ma lo è ancor di più in una edizione come questa, che si caratterizza proprio per quello che è a mio avviso il requisito precipuo di qualsiasi opera destinata a rimanere: la "problematicità". E non v"e dubbio che questa sia un'opera destinata a rimanere, nonostante i piccoli e, per la verità, più presunti difetti che ho qui creduto di ravvisare e che appaiono del tutto irrilevanti di fronte agli enormi passi in avanti che essa ha, in tutti i sensi, portato alla conoscenza di questo autore.

Domenico Cufalo

Liceo Scientifico “Ulisse Dini”, Pisa (Italy) cufalo@gmail.com 